\title{
Ring and Lens Formation
}

\author{
Françoise Combes \\ DEMIRM, Observatoire de Paris, 61 Av. de l'Observatoire, F-75014, \\ Paris
}

\begin{abstract}
The dynamical mechanism to form rings at Lindblad resonances in a barred galaxy is now well-known: due to its dissipative character, the gas is forced in a spiral structure, and experiences torques from the bar potential. Angular momentum is transferred until gas accumulates in the resonant rings. Some problems remain however to account for all observations, such as the very different time-scales for nuclear, inner and outer ring formation, while the three are frequently observed in the same galaxy; the shapes, orientations and thickness of the rings, etc... The adequacy of the present gas dynamical modelizations is discussed.

Lenses are secondary components of barred galaxies that could originate from bar evolution. No model until now has met the observational constraints, in particular the sharp edge of the lenses, their strong velocity anisotropy, and their small thickness. We propose here that lenses are the result of partial bar destruction, a necessary step in a feedback cycle of bar formation-destruction, a cycle driven by gas accretion.
\end{abstract}

\section{Introduction}

Rings are a precious tool in our understanding of barred galaxies, since they delineate the resonances, which tell us about the angular pattern speed of the bar. Their morphology (pseudoring or well developed, elongated or not) provides information about the strength of the pattern, and its age. The theory of ring formation is well developed, but is based on gas dissipation that is still not well known; rings do not form in galaxy models where the gas component is considered as a continuous fluid, with shocks and large dissipation; the various gas dynamical models are discussed in section 2 .

Lenses are secondary components almost as frequent as rings, but have not attracted much theoretical attention. They occur essentially in barred galaxies, and are then correlated in size and orientation to the bar. They could also inform us about the precise pattern speed of the bar, and also on its secular evolution. A formation mechanism is proposed in section 3 , supported by $\mathrm{N}$-body simulations. Recent review articles on these topics can be found in Martinet (1995), Combes (1993) and Buta \& Combes (1995). 


\section{Rings}

\subsection{Observational Clues}

Well defined rings are found in $25 \%$ of spiral galaxies, and pseudorings in another $33 \%$. Rings are preferentially found in barred galaxies, and are then aligned with the bar (Buta 1992). I refer to the beautiful presentation by Ron Buta (this meeting) for the definition of the various rings. An important aspect to check formation theories is that gas is always present in rings, although earlytype galaxies have more frequently rings (late-types have mainly pseudorings). Rings are the site of enhanced star-formation, and are conspicuous in $B-I$ images (e.g. Buta \& Crocker 1991, 1993). These observations support a gas dynamical origin for the rings. Rings are sometimes observed in non-barred galaxies. However, there might exist oval distortions hard to see in the optical domain, and that are revealed in the infrared (Zaritsky \& Lo 1986, McLeod \& Rieke 1995). It is interesting to stress that the three kind of rings (nuclear, inner, outer) are sometimes observed simultaneously in the same galaxy (e.g. IC 1438, NGC 1433, NGC 7217, etc..). This is an important constraint for the models.

\subsection{Formation Mechanisms}

The widely accepted theory is that rings are formed by gas accumulation at resonances, under the influence of the gravitational torques exerted by the bar potential on the gas. Nuclear rings correspond to ILR, inner rings to the ultraharmonic resonance (UHR) or $4 / 1$ resonance, near corotation, and the outer rings to OLR, as confirmed by the observed ratios between the different radii (cf. Athanassoula et al. 1982, Buta 1986). Bisymmetric tangential forces are exerted on the gas in addition to radial forces, since the potential is nonaxisymmetric, with an $m=2$ perturbation. If the gas is evolving on circular orbits, these forces cancel out by symmetry, and no net torque is produced. This is also the case for elongated orbits aligned parallel or perpendicular to the bar. On the contrary, when the gas is distributed in a spiral structure, orbits are inclined with respect to the symmetry axis of the bar. This results in a net torque on the gas, which is schematically represented in Figure 1.

Due to the underlying orbital structure (e.g. Contopoulos \& Grosbol 1989), the torque changes sign at each resonance, where the spiral turns by $90^{\circ}$ : between the ILR and corotation, the torque is negative, and positive outside CR up to OLR. These torques tend to depopulate the corotation region, and to accumulate gas towards the Lindblad resonances, in a ring morphology. The gas orbits in the rings are aligned with the symmetry axis of the bar, and no net torque is acting on them. Schwarz $(1981,1984)$ was the first to demonstrate the efficiency of these gravity torques to form rings, via numerical simulations. He developed a sticky particle code, where gas clouds are test particles in a fixed barred potential, moving like free particles, except when their trajectories cross and inelastic collisions are introduced. The reduced viscosity of the code allows to see gas being driven outwards, from CR to OLR, instead of being dominated by viscous torques, that would have driven all the gas towards the center, irrespective of its location in the galaxy. 
A very convincing proof of that mechanism is the morphology of the rings themselves: they follow the shape of the periodic orbits, (for example the very characteristic dimples for the outer rings, Buta \& Crocker 1991) and at the OLR, there exist in some galaxies the two kinds of rings $R_{1}^{\prime}$ and $R_{2}^{\prime}$, perpendicular just inside OLR and parallel just outside OLR, respectively. The time-scale for the formation of outer rings is quite long, being proportional to the rotation period at the concerned radii, i.e. of the order of a few $10^{9} \mathrm{yrs}$. Of course, this time-scale is longer for a weaker bar, with weaker torques.

The inner ring forms in a shorter time-scale, corresponding to gas accumulated at the UHR resonance (4/1) just slightly inside corotation. In its motion towards the ILR due to the bar torque, some gas can stop at the UHR, where the orbits are parallel to the bar. The symmetry of the orbit then shields it from the torques. Evidence that this is the right interpretation comes from the shape of the inner rings, that are frequently squarish, or four-cornered (cf NGC 1433, Buta 1986).

Nuclear rings are even more easily formed, since the time-scale is very short: a few $10^{7}$ to $10^{8}$ yrs. They require the existence of at least one ILR. But various shapes can be obtained, according to the assumed pattern speed of the bar: the nuclear ring can be parallel to the bar, tilted in the leading direction, or even perpendicular to the bar (Schwarz 1984, Combes \& Gerin 1985).

In the long run, the inner rings are depleted to the benefit of nuclear rings. The persistence of inner rings in observed galaxies suggests that fresh gas is continuously provided to the galaxy, either from the gas released from high mass star formation, or by continuous infall of external gas.

When there exist two ILRs, the gas accumulates at the inner ILR, which can be very close to the center (inside $1 \mathrm{kpc}$, typically). The torque is negative in between the two ILRs, because the transient spiral structure formed there in the gas is leading. This can be understood from the behavior of the $\Omega-\kappa / 2$ curve, which represents the precession rates of the $m=2$ elongated orbits; outside the ILR region, the curve is decreasing with radius, so that the gas clouds losing a little bit of energy during a collision decrease their orbital radius, and increase their precession rate: they lead the stellar orbits, and are spread in a trailing spiral structure (see Figure 1). Now the $\Omega-\kappa / 2$ curve has its maximum in between the two ILRs, and then the opposite occurs, the gas after a collision will precess less rapidly, and will trail the stellar orbits. The spiral structure is leading. In fact, the transient spiral that forms between the two ILRs is a mixture of leading and trailing waves, and close to the inner ILR, the leading one dominates. The gas distribution, which is very concentrated in the center, favors the leading wave, and the dominant torque is negative.

The presence of two ILRs, in shifting the gas response with respect to the stellar bar, enhances the negative gravity torques on the gas. This considerably enhances the nuclear gas flows (Combes 1993). We can note however that the torque inside the inner ILR is still positive, and gas is not driven right into the center to fuel the nucleus. This can only occur through either viscosity (which could take a larger importance, given the small scales), or dynamical friction of giant molecular clouds on the bulge stars. When the mass concentration is high enough in the center, another pattern can develop inside the nuclear ring, as developed by Friedli (these proceedings). 


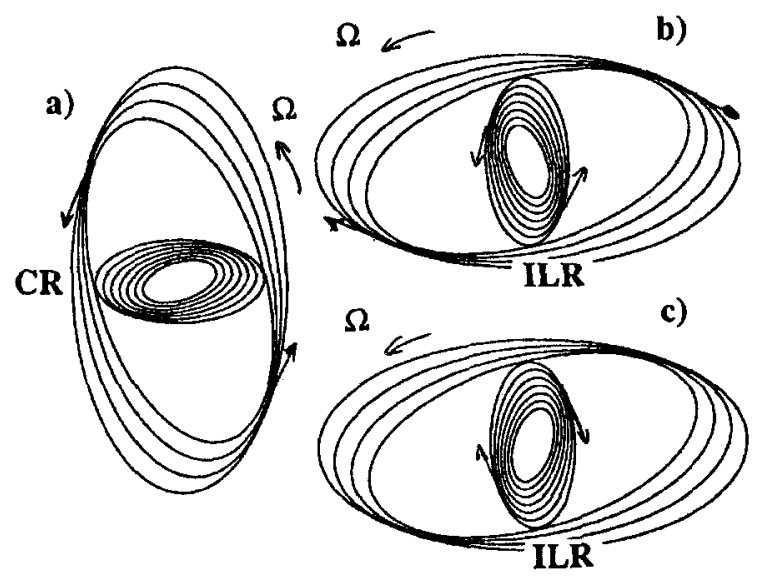

Figure 1. Schema of some gas trajectories, tilted by collisions from the general trend of periodic orbits, in a horizontal-bar potential: a) the gas leads the stars, and is spread in a trailing spiral structure, the bar torque is positive outside $C R$; b) same inside CR, but the torque now is negative, then positive inside ILR; c) near the inner ILR, the gas now trails the stars, and forms a leading spiral structure, the torque is then negative inside the outer ILR.

\subsection{Gas modelization}

Gas dissipation is a key factor in the ring formation mechanism, although viscous torques are negligible versus the gravity torques (Combes et al. 1990). Dissipation is there to cool down the gas clouds, and collisions act to deviate their trajectory to form the spiral structure. But only a few collisions per rotation are necessary, and that alone will be insufficient to drive the gas inwards in a Hubble time. We do not know precisely the actual viscosity of the interstellar medium (ISM), but given its very complex small-scale structure, it is not the point here to model it in any accurate manner. Any large-scale hydrodynamical simulation can reproduce the main characteristics of gas flow in galaxies, provided that viscous torques are negligible.

The most straightforward modelization of the ISM is to represent it as a continuous diffuse fluid, essentially governed by pressure forces. This simple model is based on the large-scale atomic gas observations (HI line at $21 \mathrm{~cm}$ ), although this quite diffuse phase appears to be composed of individual clouds at small-scale (see Burton 1992 for a review). In fact, the ubiquity of the HI component is the source of heavy blend and saturation, that makes the HI emission appear pervasive and continuous, washing out the discrete, cold and dense, hydrogen clouds. Another view of the ISM is gained from molecular cloud studies, essentially from the $\mathrm{CO}$ line at $2.6 \mathrm{~mm}$, tracing the $\mathrm{H}_{2}$ molecule that has no radio rotational line for symmetry reasons. Molecular clouds are highly clumpy, and show that most of the mass of the ISM has a very low volume filling factor. Current views of the ISM are therefore based on self-similar structures, a hierarchy of clouds over several orders of magnitude in scale (Falgarone et 
al. 1992; Pfenniger \& Combes 1994). These clouds are supported by gravity, and pressure forces could then concern only the smallest scale, much below the parsec size. There coexist several phases in the ISM, with different densities and temperatures, from the cold molecular component $(10 \mathrm{~K})$, the warm neutral and ionized phases $(8000 \mathrm{~K})$, to the hot coronal gas $\left(10^{6} \mathrm{~K}\right)$ as emphasized by McKee \& Ostriker (1977). However, most of the mass appears to be by far in the cold phase, and a view based on slightly dissipative ballistic clouds emerges. Energy is poured in this medium from the nuclear energy released in stars: supernovae explosions, stellar winds, bipolar flows, etc...

The continuous gas picture has been also favored by the observation of conspicuous dust lanes along spiral arms and bars. These very narrow features, where gas and dust appear to be compressed and the density enhanced, are highly suggestive of shocks. It is not likely, however, that large-scale shocks concern a high mass fraction of the ISM. The only fluid phase, the coronal gas, is much too hot to be affected by shocks. The ensemble of molecular clouds could act as a fluid, with clouds acting as "molecules" (Cowie 1980), but the collisional time-scale is larger than the crossing time of spiral arms, and the ensemble does not really act as a fluid (Casoli \& Combes 1982).

The first attempts to simulate gas in barred galaxies were done with the continuous fluid model (e.g. Sanders \& Huntley 1976, with the beam scheme). The simulations were not self-gravitating. Other continuous gas models have been widely used, including or not self-gravity: SPH, finite difference, fluxsplitting second order scheme (Schempp 1982, van Albada 1985, Athanassoula. 1992, Wada \& Habe 1992, Friedli \& Benz 1993, Heller \& Shlosman 1993). These modelizations are quite viscous, depending on the spatial resolution, and in particular artificial viscosity is added, in order to treat shock waves: the shock has to be spread over a few resolution cells. The dissipation can then affect the results of simulations, departing from the actual observations. In particular, the continuous-fluid simulations do not form contrasted rings, as are currently observed in barred galaxies. Rings at OLR are impossible to form in the presence of viscous torques, since the latter always drive the gas inwards, while the gravity torques are there positive outside $\mathrm{CR}$ and drive the gas outwards in the outer rings. The gas in the fluid picture is flowing so quickly towards the center, due to the viscous torques, that it does not see the resonances. The continuous-fluid approach, on the contrary, reproduces very well the shocks of the warm diffuse phase (e.g. Athanassoula 1992), which is closer to a perfect isothermal gas.

The other modelization used in galaxy hydrodynamics is the sticky particles approach, where an ensemble of gas clouds move in ballistic orbits and collide, without extra pressure and viscosity terms (Schwarz 1984, Roberts \& Hausman 1984, Combes \& Gerin 1985, Palous et al. 1993, Byrd et al. 1994). In this frame, contrasted rings are quite easy to form, and in particular the long-term behavior is very realistic, with the formation of outer rings, but there are no large-scale shocks. We can note that the SPH modelization, that considers individual particles with a finite size, is somewhat intermediate between the two modelizations: it will approach the fluid behavior only asymptotically for large particle numbers.

The differences between the two gas modelizations are drastically amplified when self-gravity is taken into account. In the fluid picture, the fast infall of gas towards the center then modifies the whole dynamics, in creating large 
central mass concentrations. The gas infall can destroy the bar very quickly (e.g. Friedli \& Benz 1993). Using different codes, simulations started with the same initial conditions give totally different results (Junqueira \& Combes 1995). The consideration of star-formation is another process that could stop or delay gas infall (Heller \& Shlosman 1993).

Given these difficulties, it can be understood that the simpler non-selfgravitating simulations give for the moment the best fit to observations. Byrd et al. (1994) with a sticky particle code have undertaken a series of simulations, trying to compare one by one the results with observed galaxies. They found that outer rings (mainly $R_{2}^{\prime}$ ) are more easily produced with fast bars (since there is then enough matter at OLR). Inner rings have generally the shape of pointy ovals (corresponding to the $4 / 1$ orbits). In the slow bar regime, nuclear rings and $\mathrm{R}_{1}^{\prime}$ ) outer rings are favored. A statistical comparison with observations reveals that the slow bars are much more frequent.

\section{Lenses}

\subsection{Observational Constraints}

Lenses are slightly elliptical features, of nearly constant surface brightness, with a sharp outer edge, and steep outer gradients. Typical examples can be seen in NGC 1553 (Freeman 1975), NGC 1291 (Kormendy 1982a) and NGC 2775, 3504, and 3945 (Kormendy 1977, 1984, Sandage \& Brucato 1979). Lenses appear to be as flat as disks, they are more 2D than 3D structures (Tsikoudi 1980, Burstein 1979). They are quite hot in the central parts, even hotter than the bulge. But they are cold at the edge, which explains their sharp cut-off (Kormendy 1984).

Lenses are closely associated with bars; Kormendy (1979) has found that $\approx 54 \%$ of SB0-a galaxies have lenses. They are especially common in early-type barred galaxies. When the bar and lens occur in the same galaxy, the bar fills the lens in one dimension ( $\theta$-like shape). The statistics on the sizes gives an average ratio of lens to inner ring sizes $L / r=1.3$, while the outer ring to lens size ratio $\mathrm{R} / \mathrm{L}$ peaks at 1.8 (Athanassoula et al. 1982). This suggests that the lens edge is near corotation. It is interesting to note that the edge of the lens is generally enhanced as with a ring.

\subsection{Formation mechanisms}

A few mechanisms have been proposed to explain the lens component, but none has been developed enough to meet all observational constraints. Kormendy $(1979,1982 \mathrm{~b})$ suggests that lenses are secondary components originating from the interaction between the bar and the bulge. If the bulge is rotating, some angular momentum can flow from the bulge to the bar, which destroys it (the bar is a wave with negative angular momentum). The secular evolution of a bar to a more axisymmetric state could form the lens. Bosma (1983) proposes that the lens is a primary component forming early; the sharp cut-off would then be a star-formation threshold. Athanassoula (1983) suggests that lenses form just like bars, through gravitational instabilities; the difference in eccentricities would come from the initial conditions, lenses forming out of a much hotter population. There remains to be found why two distinct populations of stars 
exist initially in the disk, why the edge of lenses are so sharp and cold, and why the lens components are still as flat as disks.

I will develop here another hypothesis, that the lenses are secondary components coming from the regulation of the bar strength due to a central mass concentration. Much work has been already done on the influence of a central mass concentration (or black hole, BH) on the bar secular evolution (Norman et al. 1985, Hasan \& Norman 1990, Pfenniger \& Norman 1990, Nishida \& Wakamatsu 1993, Hasan et al. 1993). When the BH scatters particles at a velocity

$$
v \approx\left(G M_{b h} / a_{b h}\right)^{1 / 2} \approx V_{r o t}
$$

then particles can escape the center and be redistributed, their orbit becomes stochastic for high enough BH masses. The bar favors clongated orbits in the center, with less angular momentum, which are more sensitive to the central BH than circular orbits. Hasan \& Norman (1990) have computed the percentage of regular $x_{1}$ orbits building the bar, as a function of the $\mathrm{BH}$ mass. This percentage falls significantly when the central mass reaches $5 \%$ of the total mass: then the percentage of stochastic orbits is so large that the bar is weakened, then destroyed for a mass fraction of 10\%. Nishida. \& Wakamatsu (1993) have shown through $\mathrm{N}$-body simulations that a bar still forms with the same amplitude in a galaxy with a mass concentration as high as $5 \%$, but its strength weakens in the long run, while a bar with no central mass concentration keeps its amplitude in a steady state through a Hubble time.

The necessary mass concentrations $(5 \%)$ to destroy the bar would correspond to a $5 \times 10^{9} \mathrm{M}_{\odot}$ black hole, which is supposed to be hosted by AGN or quasars, but not in normal spiral galaxies. But could the concentrated bulges be massive enough to play the same role? The order of magnitude given above concerning the scattered velocities leads to a required $(M / r)$ ratio comparable for the bulge and the disk, i.e. $(M / r)_{b} \approx(M / r)_{d}$, or in terms of the surface densities $\mu_{b} \approx 10 \mu_{d}$, if we assume a scalelength an order of magnitude lower for the bulge. In terms of magnitude, if the central surface brightness of disks is around $21.6 \mathrm{mag} \operatorname{arcsec}^{-2}$, that of the bulge should be at least $19.1 \mathrm{mag} \operatorname{arcsec}^{-2}$, which is sometimes observed in early-type galaxies. So the bar could indeed begin to be destroyed by the central mass concentrations in some galaxies. An effective mass concentration can also be provided by strong gas infall, since the scalelength ratio is then high (the disk to bulge scalelength ratios are usually only $\approx 4)$.

We can think of a self-regulated process, based on gas accretion: the torques due to the strong initial bar drive the gas inwards in a short time-scale. This increases the central mass concentration, up to the point where the bar weakens; there are then two ILRs and the aligned $x_{1}$ regular orbits are less preponderant. The torques weaken and the gas infall is slowed down or halted; the gas coming from the outer parts of galaxies has now time to form stars in the disk, which re-establishes the mass balance between the nucleus and the disk. When the central mass concentration is so diluted, gravitational instability can form a new bar, and the cycle is closed. To have several such cycles in a Hubble time requires however a substantial mass accretion, i.e. important gas reservoirs in the outer parts of galaxies. Another possible loop of the cycle is provided by 

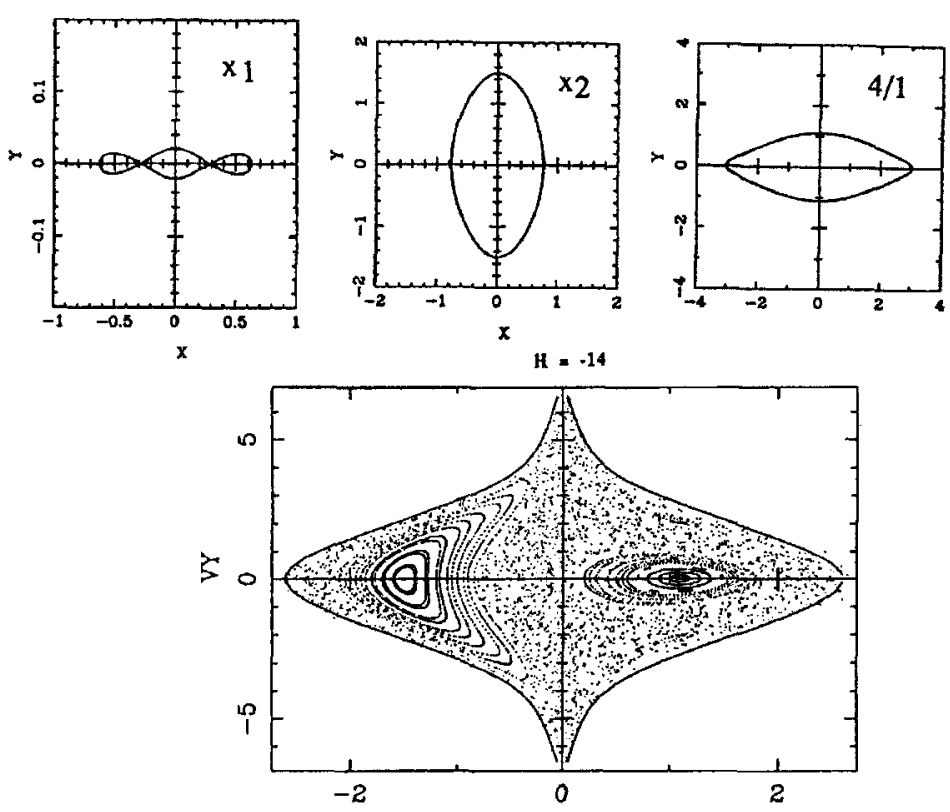

Figure 2. Some of the remaining periodic orbits in a barred galaxy with $8 \%$ central mass, and a surface of section showing the extension of chaotic orbits

the decoupling of a second bar (Friedli \& Martinet 1993), since it can delay for a while the gas infall (Combes 1994).

The consequence of this feedback cycle is that in evolved bars, there are always just about 2 ILRs. What happens to orbits in this bar regulation process? Figure 2 displays some characteristic periodic orbits that remain in the presence of a central mass concentration of $8 \%$, while a surface of section shows the extent of chaotic orbits. Near the mass concentration, the $x_{2}$ family is predominant, becoming round with increasing mass (the potential then becomes axisymmetric in the center). Then comes a region of chaotic orbits, followed near the end of the bar by a region still dominated by the $x_{1}$ elongated orbits (with lozenge shapes near the 4/1). Once in the chaotic sea, the orbits are bounded only by their limiting energy curve, in the rotating frame, where the potential is $\Phi(r)-1 / 2 \Omega^{2} r^{2}$, so that outside corotation, there is no bound. Moreover, most orbits between CR and the $-4 / 1$ resonance are stochastic (Contopoulos et al. 1989). For the ensemble of chaotic orbits inside CR, the corotation region acts as a boundary. This could be the origin of the sharp cut-off of the lens, if we consider that the chaotic orbits due to the destruction of the bar by the central mass concentration contribute in a large part to the lens component.

The edge of the lens is also delineated by a ring of particles still in regular $x_{1}$ orbits at the end of the bar. This scenario has been checked through test-particle simulations, where initial conditions include a strong central mass concentration 

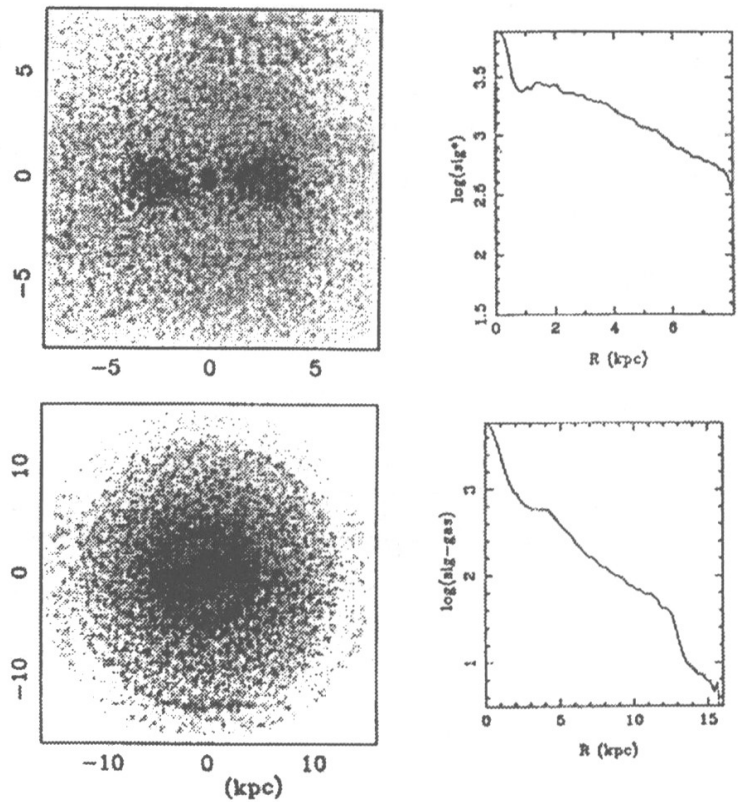

Figure 3. Results of the test-particle simulations in a barred potential with a central mass concentration of $1 \%$ for the stellar component (top) and for the gas (bottom), together with the corresponding surface density law.

(from 0 to $8 \%$ of the total mass), and where a bar is slowly introduced in the potential (Figure 3).

Test-particle simulations These simulations have been run with a Kalnajs potential for the bar (Kalnajs 1976); we have investigated the behavior of the stellar component and the gas component, the latter being treated as collisional clouds (Combes \& Gerin 1985). In the stellar component, empty regions develop in the bar, between the remaining regular orbits regions, and the bar takes the form of "ansae" for a value of the central mass of a few \% (see Figure 3). In the gas component (and certainly in the young stars formed) collisions scatter particles back in the chaotic region, and the surface density profile looks more like a plateau. At the end of the bar, the gas accumulates in a wide ring, which is not a resonant ring, but corresponds to the region of the last remaining periodic orbits.

$N$-body simulations Completely self-consistent simulations with stars and gas are shown in Figure 4. The central mass concentration is $1 \%$ and the total gas mass is $0.5 \%$ of the total mass. A very thick oval forms through dissolution of the stellar bar, with the "ansae"-shape. The gas bar is thinner and more complete. This half-destroyed bar remains for a $2 \times 10^{9}$ yrs time-scale. The luminosity profile of such a disk presents the characteristic of a typical lens. 

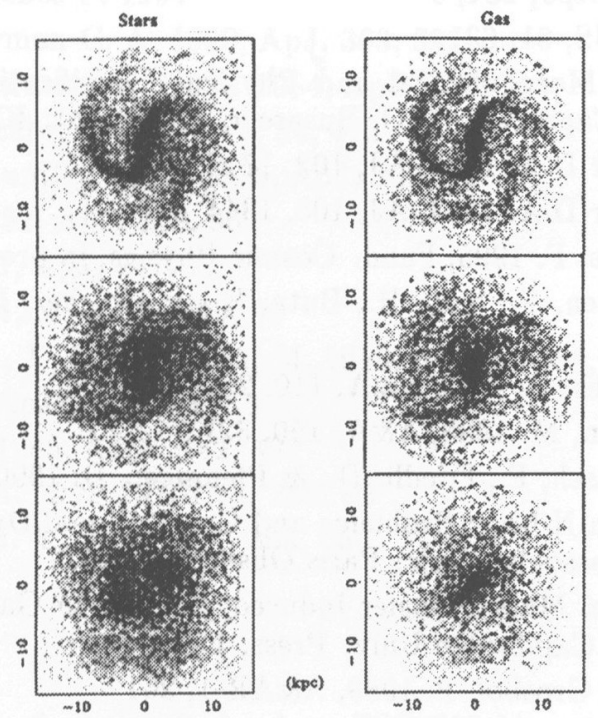

Figure 4. $\quad \mathrm{N}$-body simulations with stars and gas, starting with axisymmetric conditions, and a mass concentration of $1 \%$. The bar stays half-dissolved for a time-scale of Gyrs. Note the "ansae" in the stellar distribution.

\section{Conclusion}

We suggest that the lens component forms from a partial dissolution of the bar by a central concentration, which can be provided by gas infall. Gas accretion is stopped by the dissolution of the bar, leading to a bar-regulation cycle, where the bar-lens morphology is a particular phase. The lens boundary is corotation, its shape could be slightly eccentric (according to the shape and strength of the bar). The ring at the end of the lens is not a resonant ring, as the characteristic rings of barred galaxies. A stellar bar partially dissolved by a central mass concentration can present "ansae" at its end (4/1 periodic orbits), and a nuclear ring (or nuclear bar, $x_{2}$ orbits).

\section{References}

Athanassoula, E. 1983, in Internal Kinematics and Dynamics of Galaxies", E. Athanassoula, Dordrecht:Reidel, 243

Athanassoula, E. 1992, MNRAS, 259, 345

Athanassoula, E., Bosma, A., Créze, M., \& Schwarz, M.P. 1982, A\&A, 107, 101

Bosma, A. 1983, in Internal Kinematics and Dynamics of Galaxies, E. Athanassoula, Dordrecht: Reidel, 253

Burton, W. B. 1992, in The Galactic Interstellar Medium, D. Pfenniger \& P. Bartholdi, New York: Springer-Verlag, 1 
Burstein, D. 1979, ApJ, 234, 829

Buta, R. 1986, ApJS, 61, 631

Buta, R. 1992 in Morphological and Physical Classification of Galaxies, G. Longo, M. Capaccioli, \& G. Busarello, Dordrecht: Kluwer, 1

Buta, R. \& Crocker D.A. 1991, AJ, 102, 1715

Buta, R. \& Crocker D.A. 1993, AJ, 105, 1344

Buta, R. \& Combes, F. 1995, Fund. Cosmic Physics, in preparation

Byrd, G., Rautiainen, P., Salo, H., Buta, R., \& Crocker, D.A. 1994, AJ, 108, 476

Casoli, F. \& Combes, F. 1982, A\&A, 110, 287

Combes, F. \& Gerin, M. 1985, A\&A, 150, 327

Combes, F., Debbasch, F., Friedli, D., \& Pfenniger, D. 1990, A\&A, 233, 82

Combes, F. 1993, in N-body Problems and Gravitational Dynamics, F. Combes \& E. Athanassoula, Paris: Paris Observatory, 137

Combes, F. 1994, in Mass-Transfer Induced Activity in Galaxies, I. Shlosman, Cambridge: Cambridge Univ. Press, 170

Contopoulos, G. \& Grosbol, P. 1989, A\&AR 1, 261

Contopoulos, G., Gottesman, S. T., Hunter, J. H., \& England, M. N. 1989, ApJ, 343,608

Cowie, L. L. 1980, ApJ, 236, 868

Falgarone, E., Puget, J.-L., \& Perault, M. 1992, A\&A, 257, 715

Freeman, K. C. 1975, in Dynamics of Stellar Systems, A. Hayli, Dordrecht: Kluwer, 367

Friedli, D. \& Benz, W. 1993 A\&A, 268, 65

Friedli, D. \& Martinet, L. 1993, A\&A, 277, 27

Hasan, H. \& Norman, C. 1990, ApJ, 361, 69

Hasan, H., Pfenniger, D., \& Norman C. 1993, ApJ, 409, 91

Heller, C. H. \& Shlosman, I. 1994, ApJ, 424, 84

Junqueira, S. \& Combes, F. 1995, A\&A, in preparation

Kalnajs, A. J. 1976, ApJ, 205, 745

Kormendy, J. 1977, ApJ, 214, 359 (lenses)

Kormendy, J. 1979, ApJ, 227, 714

Kormendy, J. 1982a, in Morphology and Dynamics of Galaxies, L. Martinet \& M. Mayor. Sauverny: Geneva Observatory, 113

Kormendy, J. 1982b, ApJ, 257, 75

Kormendy, J. 1984, ApJ, 286, 116 (NGC 1553)

Martinet, L. 1995, Fund. Cosmic Physics 15, 341

McKee, C. F. \& Ostriker, J. P. 1977, ApJ, 218, 148

McLeod, K. K. \& Rieke G. H. 1995, ApJ, 441, 96

Nishida, M. T. \& Wakamatsu, K. 1993, PASJ, preprint

Norman, C. A., May, A., \& van Albada, T. S. 1985, ApJ, 296, 20

Palous, J., Jungwiert, B., \& Kopecky, J. 1993, A\&A, 274, 189 
Pfenniger D. \& Combes F. 1994, A\&A, 285, 94

Pfenniger D. \& Norman C. A. 1990, ApJ, 363, 391

Roberts, W. W. \& Hausman, M. A. 1984, ApJ, 277, 744

Sandage, A. \& Brucato, R. 1979, AJ, 84, 472

Sanders, R. H. \& Huntley, J. M. 1976, ApJ, 209, 53

Schempp, W. V. 1982, ApJ, 258, 96

Schwarz, M. P. 1981, ApJ, 247, 77

Schwarz, M. P. 1984, MNRAS, 209, 93

Shlosman, I., Frank, J., \& Begelman, M. 1989, Nature, 338, 45

Tsikoudi, V. 1980, ApJS, 43, 365

van Albada, G. D. 1985, A\&A, 142, 491

Wada, K. \& Habe A. 1992, MNRAS, 258, 82

Zaritsky, D. \& Lo K. Y. 1986, ApJ, 303, 66

\section{Discussion}

R. Buta: The nearly face-on spiral galaxy ESO 565-11, a prototype of a "misaligned bar-inner ring" galaxy, is also a misaligned bar-lens galaxy. The lens and bar axes are misaligned by $60^{\circ}$. How do you interpret such a configuration? The galaxy has no major companions.

$F$. Combes: In the simulations, the bar and lenses are aligned, except in the disturbed period of complete bar dissolution. So I do not see how this misalignment can occur without an external perturbation, such as the passage of a companion. It could be that a small companion has just merged, or a bigger companion has flown by in a hyperbolic orbit.

$J$. Kenney: The nuclear rings seen in optical images of galaxies are rings of star formation, not rings in the gas. The gas distributions are more complex than the star-forming rings. There is gas observed outside the rings, leading into it, and gas inside the rings in some galaxies, which is not forming stars. In order to determine how dissipative the ISM is, shouldn't one compare the results of ISM simulations with the distributions of gas rather than the distribution of star-formation?

F. Combes: I agree, star-formation enhances considerably the contrast of the rings. But star-formation is proportional to a certain power of the gas density (Schmidt law), in a slightly non-linear way, so that the gas density must have been maximum in the ring too. If the gas has time to accumulate in rings and form stars there before flowing inwards due to viscosity, then it is not so dissipative. The results of the ISM simulations should be compared to the observed distribution of both young stars and gas, proportionally to their mass; more ideally, the models should take into account star formation (but the processes are not yet well known).

J. Palous: Why should the dissipation be as small as possible? Is it due to lack of knowledge of the microphysics? 
$F$. Combes: I mean that nowadays gas simulations are far from achieving the right spatial resolution to represent accurately the true low viscosity of the ISM (which is clumpy down to AU-scales). With a typical resolution of $100 \mathrm{pc}$, the simulations always overestimate the viscous torques. This does not matter too much, as soon as the latter are negligible with respect to gravity torques, that's why the dissipation should be kept as small as possible. But it is also true that our knowledge of the ISM small-scale structure and its microphysics is still in its infancy. 